\title{
ㄷำ1
}

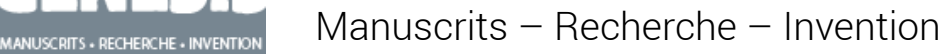

36 | 2013

Proust, 1913

\section{Proust deux mille treize}

Nathalie Mauriac Dyer

\section{OpenEdition}

\section{Journals}

Édition électronique

URL : https://journals.openedition.org/genesis/869

DOI : $10.4000 /$ genesis. 869

ISSN : 2268-1590

\section{Éditeur :}

Presses universitaires de Paris Sorbonne (PUPS), Société internationale de génétique artistique littéraire et scientifique (SIGALES)

\section{Édition imprimée}

Date de publication : 15 juin 2013

Pagination : $9-13$

ISBN : 978-2-84050-893-9

ISSN : $1167-5101$

\section{Référence électronique}

Nathalie Mauriac Dyer, «Proust deux mille treize », Genesis [En ligne], 36 | 2013, mis en ligne le 23 septembre 2015, consulté le 11 janvier 2023. URL : http://journals.openedition.org/genesis/869 ; DOI : https://doi.org/10.4000/genesis.869 


\section{Proust deux mille treize}

$A$ lors que, le 29 mai 1913, le public du tout nouveau théâtre des Champs-Élysées se déchaîne à la première du Sacre du printemps, Proust est probablement chez lui, en train de corriger les placards d'un premier volume qui a failli s'appeler «Le Temps perdu » ou encore "Charles Swann ». L'année suivante, il se flattera, sous couvert d'anonymat, qu'un même critique ait pu distinguer à la fois le Sacre et Du côté de chez Swann, qu'il définit comme faisant partie des "plus curieuses productions de la littérature et de la musique " contemporaines ${ }^{1}$. Un siècle après sa publication, Swann reste sans conteste le volume le plus lu et le plus célèbre d'À la recherche du temps perdu. Son centenaire, qui coïncide avec un autre anniversaire important - le cinquantenaire du fonds Proust de la Bibliothèque nationale de France - est l'occasion de faire le bilan prospectif d' une recherche génétique proustienne en plein essor, et de revenir sur une année cruciale dans la genèse d'un des romans majeurs du XXe siècle.

Ces manuscrits où s'est jouée l'aventure d'une genèse hors du commun, Bernard de Fallois fut le premier, au tournant des années cinquante, à s'y immerger totalement et avec passion. Pour la première fois il a accepté de revenir sur cette plongée de plusieurs années d'où il ramena deux trésors inédits, Jean Santeuil puis Contre Sainte-Beuve, substituant ainsi à l'image d'un Proust dandy et mondain celle du travailleur infatigable, et établissant les piliers de toute recherche ultérieure sur la genèse de la Recherche.

En 1962, dix ans après la publication de Jean Santeuil, les manuscrits de Proust entraient à la Bibliothèque nationale. Inventoriés et classés par Florence Callu, restaurés, ils n'étaient alors accessibles qu'à la table de la Réserve du département des Manuscrits, rue de Richelieu, tandis qu'au Centre d'Analyse des Manuscrits de la rue d'Ulm, ancêtre de l'ITEM, quelques pionniers commençaient (sur de simples microfilms en noir et blanc) la cartographie systématique de cet immense continent. Guillaume Fau, aujourd' hui en charge du fonds à la BnF, raconte l'histoire de sa constitution et de sa préservation. La dernière péripétie n'est pas la moins palpitante : c'est celle de la numérisation, suivie de la publication sur Gallica, à partir de 2009, de plus d'une centaine de cahiers et de carnets, sans compter quelques riches reliquats ${ }^{2}$. L'étape est capitale : sur l'écran de notre ordinateur ou de notre tablette, cette nouvelle salle de consultation virtuelle est ouverte,

1. Voir Corr., t. XIII, p. 161, et p. 162 la note 3 de Ph. Kolb.

2. Voir l'ensemble : <www.item.ens.fr/index.php?id=578147>. 
vingt-quatre heures sur vingt-quatre, partout dans le monde, offrant des images couleur en haute définition qu' il est possible d'agrandir et de télécharger. La recherche sur l' 'auve de Proust en est profondément démocratisée et bouleversée.

Pourtant il faut un fil d'Ariane dans ce dédale, ou un Virgile dans ces cercles infernaux de pages surchargées d'ajouts et de paperoles. Le manuscrit «brut » doit être rendu lisible (par une transcription) et intelligible (par un commentaire qui le situe dans l'ensemble des autres manuscrits, et qui en élucide les obscurités) - autrement dit il doit être édité : la collection des Cahiers 1 à 75 de la Bibliothèque nationale de France 3 est aujourd' hui ce vaste chantier (et ce défi majeur). Dirk Van Hulle revient sur les trois premiers cahiers publiés et situe l'originalité de l'entreprise dans une réconciliation entre tradition philologique et édition génétique : il s'agit de relever le plan d'une création labyrinthique, ramifiée sur près de quinze ans. Deux des volumes parus, le Cahier 71 et le Cahier 54 de 1913 et 1914, donnent l'occasion de nouveaux travaux : Guillaume Perrier se penche sur quelques procédés mnémotechniques forgés par Proust pour baliser ses propres cahiers, tandis que Yuji Murakami identifie dans l' "espionnite " et l'antidreyfusisme l'origine des métaphores xénophobes et antisémites dont fait l'objet Albertine, dès lors qu'elle est soupçonnée par son ami d'être du côté de Gomorrhe.

Cette dernière étude, qui se situe, comme la grande somme récente de Kazuyoshi Yoshikawa sur Proust et la peinture ${ }^{4}$, au carrefour de la génétique textuelle et de l'histoire culturelle, n'aurait sans doute pas été possible sans la numérisation d'autres fonds essentiels à la recherche, les fonds d'imprimés et de périodiques. Gallica et archive.org nous donnent aujourd' hui accès en quelques clics à toutes, ou presque toutes, les lectures de Proust. Cette imposante mémoire de la culture, mémoire quasi absolue, est aussi celle du roman, rappelle Antoine Compagnon : et le chercheur n'y trouve son chemin que grâce à son «flair » et à son intuition. Dans l' "épaisseur mémorielle » de la Recherche, ce ne sont d'ailleurs pas les traits les plus saillants mais des détails infimes, des angles perdus, que nos interprétations recueillent, à rebours vraisemblablement des attentes de l'écrivain.

Sans doute sommes-nous attentifs aux lapsus, aux silences, aux aveux qui échappent au narrateur (comme, nous rappelle Antoine Compagnon, $M$. de Norpois l'était, cruellement, aux gaffes du jeune héros). Beaucoup, qu'ils l'admettent ou non, sont quant à eux happés, fascinés par la "vie» de l'écrivain, victimes à des degrés divers de cette condition postbeuvienne que Barthes avait baptisée "Marcellisme ». Même du côté des manuscrits, nous n'échapperons pas tout à fait au "biographique », en particulier dans sa variante épistolaire, car la genèse du roman s'écrit là aussi : depuis l'édition de référence en vingt et un volumes procurée par Philip Kolb, la correspondance est l'autre massif manuscrit incontournable des études génétiques sur l'œuvre de Proust. Françoise Leriche, " continuatrice » des travaux fondamentaux de Kolb, le rappelle, l'édition numérique est la seule solution satisfaisante pour un corpus de ce type, en constante expansion et reconfiguration (l'apparition d'une seule lettre inédite pouvant provoquer des redatations en cascade). Or ces documents, moins surchargés que les manuscrits de travail - car destinés à une communication immédiate - peuvent, sans trop de difficultés, faire l'objet des encodages qui les rendront explorables en tous sens (par correspondant, par date, par

3. Brepols Publishers et Bibliothèque nationale de France.

4. Proust et l' art pictural, Paris, Champion, 2010. 
domaine thématique...). La numérisation des quelques milliers de lettres de Proust serait ainsi, nous dit Françoise Leriche, la première " brique » d' un futur hypertexte électronique proustien qui pourrait absorber tous les matériaux et documents d'une " auvre » dont les frontières sont elles-mêmes en constante redéfinition, mais dont les manuscrits de travail restent le cour.

Quant à la numérisation de ces derniers - non pas en mode image, puisqu'elle est acquise, on l'a vu, mais en mode texte, de façon à en structurer et donc pouvoir en exploiter les contenus - elle est le préalable de toute édition électronique authentique et fait l'objet de recherches actives. Elena Pierazzo présente avec Julie André un prototype innovant d'encodage de quelques folios du Cahier 46 qui a été réalisé selon les principes de la Text Encoding Initiative (TEI). L'encodage est associé à un visualiseur diplomatique qui permet de suggérer le mouvement de l'écriture. L'édition électronique serait également le support le plus approprié à la diffusion du manuscrit morcelé d'À l'ombre des jeunes filles en fleurs, que Pyra Wise travaille patiemment à reconstituer depuis une dizaine d'années. Car les manuscrits de Proust sont dispersés, non seulement du fait des aléas de leur histoire, mais aussi en raison des nombreux découpages (suivis de collages) auxquels l'écrivain les soumettait au cours de la genèse. En témoigne le placard de Du côté de chez Swann que nous reconstituons ici virtuellement, placard dont la partie gauche se trouve aujourd' hui à la Fondation Martin Bodmer, en Suisse, et la partie droite au département des Manuscrits de la Bibliothèque nationale de France. Il faut se projeter dans l'avenir, espérons-le proche, où sur une " table de la Réserve » virtuelle, un chercheur pourra appeler toutes les richesses proustiennes de bibliothèques réunies en réseau.

On peut dire de 1913, à mi-chemin ou presque d'une longue genèse inachevée, qu'elle fut une année " capitalissime ». Proust, refusé par Fasquelle, la Nouvelle Revue Française et Ollendorff, se résout à la publication à compte d'auteur de la dactylographie du «Temps perdu » (sept cent douze pages), reçoit et corrige d'abondance les placards que lui envoie Grasset au printemps - ces grandes planches regroupant huit pages imprimées consécutives - et publie finalement, en novembre, Du côté de chez Swann, premier volume d'une trilogie, et non plus d'un diptyque comme il l'avait d'abord prévu. C'est le début d'une longue expansion que seule la mort interrompra, et que Gaston Gallimard, selon ses propres mots, "rationalisera » dans les sept tomes canoniques que nous connaissons, dont trois publiés à titre posthume.

Sur les placards, le travail de révision est spectaculaire: non seulement parce que, sous la pression de Grasset, Proust a dî en réduire le nombre de quatre-vingt-quinze à soixante et $u n^{5}$, mais parce qu'il s'y livre à d'amples réécritures, presque comme s'il s'agissait d'un nouveau manuscrit. Il barre, déplace, raboute des fragments en utilisant les trois jeux qu'il a reçus ; il ajoute, colle des béquets - de nombreux béquets, qui sont, dans l'histoire génétique du roman, les toutes premières "paperoles » après celles de la dactylographie $d u$ "Temps perdu ». Non seulement le titre du volume, mais le titre général se fixe : "Les Intermittences du cour » deviennent À la recherche du temps perdu, «Le

5. Si l'on compte les deux placards qu'il a « rapportés » après le placard 59 pour créer sa nouvelle fin. 
Temps perdu » laisse place à Du côté de chez Swann. Selon les propres mots de Proust, "Il ne reste pas une ligne sur 20 du texte primitif (remplacé du reste par un autre). C'est rayé, corrigé dans toutes les parties blanches que je peux trouver, et je colle des papiers en haut, en bas, à droite, à gauche, etc. 6 ". Les ouvriers typographes doivent recomposer et réimposer le livre "presque trois fois 7 ", travail considérable, et fort coûteux pour l'auteur. Ces placards corrigés, conservés depuis 2001 à la Fondation Bodmer - et qui, à l'initiative de Jean-Yves Tadié, font chez Gallimard à l'occasion du centenaire de Swann l'objet d'une édition en fac-similé transcrite par Charles Méla - avaient encore été peu exploités ${ }^{8}$. Dans la première partie, "Combray ", Isabelle Serça examine un bouquet de corrections convergentes qui révèlent un Proust nouvellement soucieux d'incarner, à travers la particularité de personnages ou de situations, les " grandes lois » qui font de la Recherche une cuvre dogmatique : le travail est méticuleux, systématique. Du côté $d$ " Un amour de Swann », Françoise Leriche revient sur la soirée chez la marquise de Saint-Euverte au cours de laquelle Swann réentend la "petite phrase ", désormais attribuée à Vinteuil - Vinteuil, précisément né en 1913 sur ces placards de la fusion de deux personnages secondaires, le naturaliste de Combray, Vington, et le compositeur Berget ${ }^{9}$. Françoise Leriche présente les additions qui saturent les grandes marges des placards : sous l'influence de certains concerts auxquels il a assisté au printemps de 1913, Proust y infléchit le discours du roman sur la musique, accusant d'autant plus l'esthétisme fin de siècle de Swann qu'il sait devoir ménager la découverte, par le héros seul, de la modernité de Vinteuil. Mais Proust fut-il si sensible au modernisme esthétique de l'immédiate avantguerre, en tout cas à celui des Ballets russes ? Pour ma part, je doute même qu'il ait assisté à l'autre pièce scandaleuse de la troupe de Diaghilev, L'Après-midi d'un faune, en $1913 \mathrm{ou}$ lors de sa création l'année précédente, et cela même si le "tableau chorégraphique » de Nijinsky a laissé des traces sur un des placards du premier volume (ceux qui sont conservés à la BnF, cette fois) : comme dans un cahier de 1915, c'est à travers le filtre d'une critique du journaliste Henry Bidou que Proust s'y intéresse.

C'est une cuvre en pleine métamorphose dont la Guerre interrompt la publication, même si, au début de 1913, Proust pouvait penser que les deux volumes des "Intermittences du cour », "Le Temps perdu » et "Le Temps retrouvé », auraient fini de paraître l'année suivante. Mais dès le mois d'août, quand il revient en catastrophe de Cabourg avec son chauffeur-secrétaire Alfred Agostinelli (qui le quittera en décembre et se noiera à la suite d'un accident d'avion le 30 mai 1914), le roman bascule. Proust jette dans un cahier qu'il intitule Dux les linéaments d'un drame de la jalousie et de la possession, relate à l'automne dans un autre cahier, Vénusté, les affres de $M$. de Charlus amoureux d'un jeune aviateur, revient à Dux pour raconter la fuite d'Albertine, à Vénusté l'année suivante pour le récit de sa mort accidentelle et du chagrin du protagoniste. C'est, en quelques mois, la cristallisation de ce qu'il appellera désormais, comme dans une tragédie, "l'Épisode ", et dont l'impact sur la structure la plus

6. Corr., t. XII, p. 132 .

7. Ibid., t. XIII, p. 407.

8. Voir Anthony Pugh, The Growth of À la recherche du temps perdu. A Chronological Examination of Proust's Manuscripts from 1909 to 1914, Toronto, University of Toronto Press, 2004 ; Jo Yoshida, « Ce que nous apprennent les épreuves de Du côté de chez Swann dans la collection Bodmer », BIP, n 35, 2005.

9. Voir K. Yoshikawa, « Vinteuil ou la genèse du Septuor », Cahiers Marcel Proust 9, Études proustiennes III, 1979. 
profonde du roman se fera sentir jusqu'à sa mort, en 1922. Dès novembre 1913, à peine publié, le plan en trois volumes qui accompagne Du côté de chez Swann est caduc. Mais on aurait tort de croire que le roman imite la vie - c'est bien plutôt la vie qui adopte déjà la forme romanesque d'un scénario, ou plutôt, comme le formule exemplairement le narrateur dans un cahier de la Guerre, qui mélange les "genres » : " [...] telle vie est comme un essai de psychologie subjective spontanément poursuivi, mais qui fournit à quelque distance son "action" au roman purement réaliste d'une autre réalité, d'une autre existence, et [dont] à leur tour les péripéties viennent infléchir la courbe et changer la direction de l'essai psychologique 10 ». Les études de Yuji Murakami et Guillaume Perrier sur Dux et Vénusté explorent sous des angles inattendus ce jeu de masques et de soupçons où la cruauté et la mauvaise foi de l'amoureux rivalisent avec celles des antisémites et des antidreyfusards, où des codages intimes transforment secrètement les pages d' un cahier en " tombeau».

Dans une étude qui revient sur la genèse précoce, dans le projet Contre SainteBeuve de 1909, d'un des lieux les plus fascinants de la Recherche - le long incipit de Swann, tourbillonnant dans les « mondes désorbités » du demi-sommeil -, Maya Lavault s'interroge, après bien d'autres, sur la naissance de l'écriture ou plutôt sur ce qu'on pourrait appeler la mise en scène de cette naissance : à travers des micro-récits qui apparaissent, s'apparient, disparaissent, insistent, la fiction esquisse quelques figures de son avenir et, déjà, travaille à effacer ses propres traces. Un nouveau mouvement est lancé, un mouvement dont les manuscrits, "gardiens fidèles ", conservent toutes les promesses, tous les possibles qu'il ne tient qu'à nous de déplier. Gageons que, dans moins de dix ans, à l'occasion d'un autre centenaire, celui de la disparition de l'écrivain, ce sera un autre continent manuscrit proustien, encore, que nous célébrerons.

Nathalie Mauriac Dyer

\section{Varia}

L'ombre de Proust ne nous quitte pas dans les Varia, puisque Guillaume Bellon étudie la trace qu'elle a laissée dans les derniers manuscrits de Barthes et le rôle qu' elle a joué dans l'évolution vers une nouvelle forme d'écriture dont témoignent ces manuscrits. Il expérimente au passage le traitement d'une constellation de documents génétiques hétérogènes qui ne peuvent en aucun cas être considérés comme des réécritures les uns des autres, mais qui jettent des lumières irremplaçables sur leurs genèses respectives.

Jacques Neefs revient sur la poétique de la prose flaubertienne et montre qu'en dépit de son idéal de perfection formelle, elle n'est pas issue d'une esthétique abstraite préexistante,

10. Cahier XII, paperole au folio $121 \mathrm{r}^{\circ} ; A D$, IV, p. 82. 
mais d'une recherche obstinée de l'intensité, à travers la manifestation d'un "réel écrit », qui s'impose avec toute la puissance objective d'une apparition et toute la subjectivité qui émane, comme l'avait bien vu Proust, du choix des mots et des rythmes.

En décrivant plusieurs types de manuscrits et de fichiers du zoologiste Karl von Frisch, ayant trait à ses recherches sur la fameuse "danse des abeilles », Christoph Hoffmann montre l'importance, pour la genèse de la connaissance scientifique, chez ce savant et sans doute chez bien d'autres chercheurs, de la prise de notes, des différents systèmes d'organisation de ces notes, et plus généralement de la dimension scripturale. Il étudie les processus de condensation et de réduction du foisonnement des observations expérimentales, l'organisation a priori des données et la réorganisation après coup qui conduit à la découverte par des voies imprévues.

La rubrique Chroniques II comporte des comptes rendus et, comme tous les numéros pairs de Genesis, une bibliographie des publications génétiques parues dans l'année écoulée.

La Rédaction de Genesis 